\title{
A!
}

This is an electronic reprint of the original article.

This reprint may differ from the original in pagination and typographic detail.

Pastor Figueroa, Giancarlo; Mora-Jiménez, Inmaculada; Jäntti, Riku; Caamaño, Antonio J.

\section{Constructing measures of sparsity}

Published in:

IEEE Transactions on Knowledge and Data Engineering

DOI:

10.1109/TKDE.2020.3029851

Published: 01/08/2022

Document Version

Peer reviewed version

Please cite the original version:

Pastor Figueroa, G., Mora-Jiménez, I., Jäntti, R., \& Caamaño, A. J. (2022). Constructing measures of sparsity. IEEE Transactions on Knowledge and Data Engineering , 34(8), 3643 - 3654.

https://doi.org/10.1109/TKDE.2020.3029851

This material is protected by copyright and other intellectual property rights, and duplication or sale of all or part of any of the repository collections is not permitted, except that material may be duplicated by you for your research use or educational purposes in electronic or print form. You must obtain permission for any other use. Electronic or print copies may not be offered, whether for sale or otherwise to anyone who is not an authorised user. 


\title{
Constructing Measures of Sparsity
}

\author{
Giancarlo Pastor ${ }^{\circledR}$, Inmaculada Mora-Jiménez ${ }^{\circledR}$, \\ Riku Jäntti ${ }^{\oplus}$, Senior Member, IEEE, and Antonio J. Caamaño ${ }^{\oplus}$
}

\begin{abstract}
This paper presents a rigorous but tractable study of sparsity. We postulate a definition of sparsity that is as broad as possible, so that it generates all the various measures that are useful in practice, but narrow enough that the fundamental properties of generalized sparsity still hold. As we work through the various ways of demonstrating the advantageous properties of sparsity, we illustrate its meaning from geometrical and operational perspectives. Thereafter, we construct specific measures of sparsity which are successfully qualified in complexity analysis and sparse optimization scenarios. Overall, our main objective is to construct measures of sparsity that will facilitate and enhance the design of the next innovative sensing technologies.
\end{abstract}

Index Terms-Axioms, complexity, concentration, diversity, effective, entropy, fairness, generalized convexity, inequality, information, sparsity, uncertainty

\section{INTRODUCTION}

SENSING technologies, social networks included, have Sreached a breakthrough and the newly available big data is imposing increased-complexity requirements for data engineers seeking to gain insights from the data. These requirements in storage, transmission, and other resources, have promoted the growth of the field of sparse recovery [1]. In brief, sparse recovery is the set of methods and algorithms that aim to increase the resources efficiency of large sensors, under the assumption that the information content of data is sparse in a given domain. Formally, it could be written as the optimization problem for searching the solution with the sparsest information content given few samples.

However, to find the sparsest solution is equivalent to finding the solution with fewest non-zero elements, which is NP-hard if the pseudo-norm- 0 of vectors is used. Therefore, it is common practice to use a convex proxy, the norm-1. Nonetheless, there has been an increase in applying non-convex measures [2], including the ratios of norms [3], as alternatives to the norm-1. While non-convex measures are more challenging to optimize, they outperform the norm-1 in terms of promoting sparsity [4] and may enable reconstruction of the data from substantially fewer measurements [5]. Nowadays, there are many available state-of-the-art recovery algorithms with both, convex and non-convex measures [6]. However, nonconvex measures may complicate later stages in the optimization algorithm design, commonly due to the complexity or low-speed of computing subgradients [7]. Therefore, in this

- Giancarlo Pastor and Riku Jäntti are with the Department of Communications and Networking. Aalto University School of Electrical Engineering, 02150 Espoo, Finland. E-mail: (giancarlo pastor, riku.jantti)@aalto.fi.

- Inmacudada Mora-Jiménez and Antonio J. Caamaño are with the Department of Signal Theory and Communications, Telematics and Computing, King Juan Carlos University, 28943 Madrid, Spain.

E-mail: (inmaculada mora, an tonio.caamano)@urjc.es.

Manuscript received 3 Aug. 2019; revised 10 Mar. 2020; accepted 30 Sept. 2020. Date of pultication 0.0000 ; date of current version 0.0000 .

(Corresponding author: Giancarlo Pastor.)

Recommended for acceptance by J. Tang.

Digital Object Identifier no. 10.1109/TKDE.2020.3029851 paper, we take a strategic step backward to thoroughly analyze the dynamics, properties and geometry of sparsity. Overall, our objective is to construct measures of sparsity that will facilitate and enhance the design of recovery algorithms and thus of next innovative sensing technologies [8], [9], [10].

Our main contributions are twofold:

1) A new and simplified test for sparsity qualification.

First, we formalize and package the widespread intuition about sparsity into an equivalence relation, which is a pivotal tool in mathematics [11]. Then, we use this equivalence relation to explain the complex dynamics of sparsity via a minimal characterization of sparsity. This minimal characterization provides $a$ simplified test to qualify sparsity measures. Moreover, contrary to related work, we derive a large set of fundamental properties of sparsity from this characterization (and not the other way around). One of these properties is the generalized convexity, which will suggest the existence of sparsity beyond traditional convex ones.

2) A set of sparsity-sensitive tunable measures.

The generalized convexity nature of sparsity, and advances in generalized convexity [12], [13], allow us to state sufficient conditions for ratios of functions to assess sparsity. Then, following novel ideas from sparse recovery [14], we interpret the geometry and operational meaning of these ratios or measures. The proposed measures provide comparable recovery accuracy levels in sparse recovery problems. Remarkably, the measures provide outstanding computation times in complexity analysis, qualifying them for large-scale data analysis.

The paper is organized as follows. First, Section 2 introduces the proposed framework. The framework includes basic assumptions on the sparsity behaviour and key tools to formulate the axioms of sparsity in Section 3 . We will demonstrate then the properties of sparsity from these axioms. These properties will help us to construct measures of sparsity in Section 4, and to study their geometry and 
TABLE 1

\section{Summary of Main Notation}

\begin{tabular}{|c|c|}
\hline Symbol & Description \\
\hline $\begin{array}{l}\sim_{s} \\
\propto \\
\alpha \\
{[\cdot]_{s}}\end{array}$ & $\begin{array}{l}\text { sparsity equivalence relation of vectors } \\
\text { absolute value (component-wise) of vectors } \\
\text { proportionality relation of vectors } \\
\text { equivalence class, with respect to relation } \sim_{s}\end{array}$ \\
\hline$\underset{\oplus}{\|\cdot\|_{p}}$ & $\begin{array}{l}p \text {-(pseudo-)norm of vectors } \\
\text { generalized or power } p \text {-mean of vectors } \\
\text { concatenation of vectors }\end{array}$ \\
\hline$\succ$ & majoriza tion pre-order of (real) vectors \\
\hline$\circ$ & composition of functions \\
\hline$\alpha, \beta$ & parameters of measures sott and $h_{\text {out }}$ \\
\hline$\stackrel{\eta, \omega, \omega_{g}}{\boldsymbol{\Theta}}$ & $\begin{array}{l}\text { decomposition function and weights, respectively } \\
\text { increasing function }\end{array}$ \\
\hline$\Omega_{s}^{\pi}, \Omega_{h}$ & $\begin{array}{l}\text { permutation (component-wise) of vectors } \\
\text { sparsity and entropy domains, respectively }\end{array}$ \\
\hline $\begin{array}{l}c, q_{\mathrm{q}} \\
\text { Card }\end{array}$ & $\begin{array}{l}\text { arbitrary scalars, } t=0,1, \ldots \\
\text { cardinality or dimension of vectors }\end{array}$ \\
\hline Conv & convex hull of (a set of) vectors \\
\hline$n, b$ & dimensions of $\mathbf{w}$ and $\mathbf{p}$, respectively \\
\hline$s, h$ & sparsity and entropy, respectively \\
\hline $\begin{array}{l}s_{\text {ott }}, h_{\text {ott }} \\
\text { sign } \\
S^{n}\end{array}$ & $\begin{array}{l}\text { effective sparsity and entropy } \\
\text { sign (component-wise) of vectors } \\
\text { n-dimensional unit sphere }\end{array}$ \\
\hline $\mathbf{v}, v_{i}, v_{j}$ & vector (in bold) and its components \\
\hline
\end{tabular}

operational meaning. Thereafter, in Section 5, we will qualify these measures via standard benchmarks. Finally, Section 6 will present related work and a discussion and analysis of previously proposed axioms, properties and measures.

\section{Preliminaries: Proposed Framework}

Sparsity has been traditionally defined to operate on the set $\mathbb{R}_{+}^{n}$ of non-negative real vectors [15]. In this paper, we broaden the scope and construct sparsity to operate on the entire set $\mathbb{R}^{n}$ of real vectors. We achieve this via an equivalence relation which will also be essential to formulate the axioms that characterize sparsity. Thus, the present Section starts with a brief introduction to equivalence relations. Table 1 summarizes our main notation.

A relation $\sim$ is said to be an equivalence relation [11] if and only if, for any real vectors $\mathbf{u}, \mathbf{v}, \mathbf{w} \in \mathbb{R}^{n}$,

- $\quad \mathbf{u} \sim \mathbf{u}$ (reflexive)

- $\mathbf{u} \sim \mathbf{v}$ if and only if $\mathbf{v} \sim \mathbf{u}$ (symmetric)

- if $\mathbf{u} \sim \mathbf{v}$ and $\mathbf{v} \sim \mathbf{w}$, then $\mathbf{u} \sim \mathbf{w}$ (transitive).

As a consequence, an equivalence relation $\sim$ partitions the set $\mathbb{R}^{n}$ into disjoint sets called equivalence classes, or simply classes for short. The dass of a vector $\mathbf{w}$, denoted [w], is the set of all vectors $\mathbf{v}$ which are equivalent to $\mathbf{w}$

$$
[\mathbf{w}]=\left\{\mathbf{v} \in \mathbb{R}^{n}: \mathbf{v} \sim \mathbf{w}\right\} \subset \mathbb{R}^{n} .
$$

Therefore,

- an equivalence class contains all vectors which are equivalent to each other, and thus

- a vector belongs to a unique equivalence class.

The proposed framework will build on an equivalence relation $\sim_{s}$ that gathers all vectors with the same level of sparsity into the same dass. Under this framework, we will construct sparsity as a measure $s$, which computes the sparsity of a vector $\mathbf{v}$ as the sparsity of any vector in its class $[\mathbf{w}]_{s} \ni \mathbf{v}, \quad s: \mathbb{R}^{n} \rightarrow \mathbb{R}: \mathbf{v} \mapsto s(\mathbf{v}) \equiv s(\mathbf{w})$. Furthermore, in order to facilitate computations, we will gather unique representative vectors $\mathbf{w}$ from each class into a tractable set called the sparsity domain, denoted $\Omega_{\mathrm{s}}$. Therefore, it would suffice to construct sparsity to operate on $\Omega_{\mathrm{s}}$

$$
s: \Omega_{\mathbb{s}} \rightarrow \mathbb{R}: \mathbf{w} \mapsto s(\mathbf{w}) .
$$

Our task in the next Section 2.1 is then to define that suitable equivalence relation $\sim_{s}$, and later in Section 2.2 to construct the sparsity domain $\Omega_{\mathrm{s}}$.

\subsection{Sparsity Equivalence Relation}

According to the literature [4], the sparsity of a vector $\mathbf{w}$ should depend on its components, $w_{i}, i=1, \ldots, n$, so that sparsity should be: (1) only affected by their absolute values, (2) not affected by their order, and (3) not affected by their scale. We formalize this widespread intuition regarding sparsity into the following hypothesis.

Hypothesis 1 ( $\mathrm{H} 1$ s Sparsity equivalence). For any pair of vectors $\mathbf{v}, \mathbf{w} \in \mathbb{R}^{n}$, we say that "v is sparsity equivalent to $\mathbf{w}^{\prime \prime}$, denoted $\mathbf{v} \sim_{\mathrm{s}} \mathbf{w}$, and meaning, both $\mathbf{v}$ and $\mathbf{w}$ have the same level of sparsity, if the absolute value of one vector is proportional to a permutation of the absolute value of the other

$$
\mathbf{v} \sim_{\mathrm{s}} \mathbf{w} \equiv|\mathbf{v}| \propto \pi(|\mathbf{w}|),
$$

where $\propto$ denotes "is proportional to", $|$.$| computes the absolute$ value (component-wise), and $\pi$ permutes the order of components.

Clearly, the relation $\sim_{s}$ is an equivalence relation, i.e., it is reflexive, symmetric and transitive. Then, according to $\mathrm{H} 1 \mathrm{~s}$, a vector $\mathbf{w}$ is sparsity equivalent to any vector $\mathbf{v}$ with the same (in magnitude) components but rather these:

- have different signs, or

- have been permuted, or

- have been all equally scaled.

That is, the relation $\sim_{s}$ partitions $\mathbb{R}^{n}$ into disjoint classes, and each class contains vectors with the same level of sparsity. As an example, in $\mathbb{R}^{2}$, the dass of vector $(4,3)$ contains vectors of the form

$$
\begin{aligned}
& \{(4 r, 3 r),(4 r,-3 r),(-4 r, 3 r),(-4 r,-3 r), \\
& (3 r, 4 r),(3 r,-4 r),(-3 r, 4 r),(-3 r,-4 r) ; r>0\} .
\end{aligned}
$$

Eight of these vectors (those with $r=0.2$ in (4)) are part of the unit-sphere $S^{2}$, see Fig. 1. This suggests that, the sparsity domain is one eighth of the entire $\mathbb{R}^{2}$. Moreover, in the Figure, the red thick arc outlines the potential sparsity domain. Our task in the next Section 2.2 is then to perform this packaging of unique representative vectors from each class into a tractable set.

\subsection{Sparsity Domain}

The desired behavior of sparsity, defined in (2), is captured in our main hypothesis as follows.

Hypothesis 2 (H2s Sparsity assessment). $\forall \mathbf{v} \in \mathbb{R}^{n}, \exists l w \in$ $\Omega_{\mathrm{s}}$ :

$$
\mathbf{v} \sim_{s} \mathbf{w} \quad \Rightarrow \quad s(\mathbf{v})=s(\mathbf{w})
$$




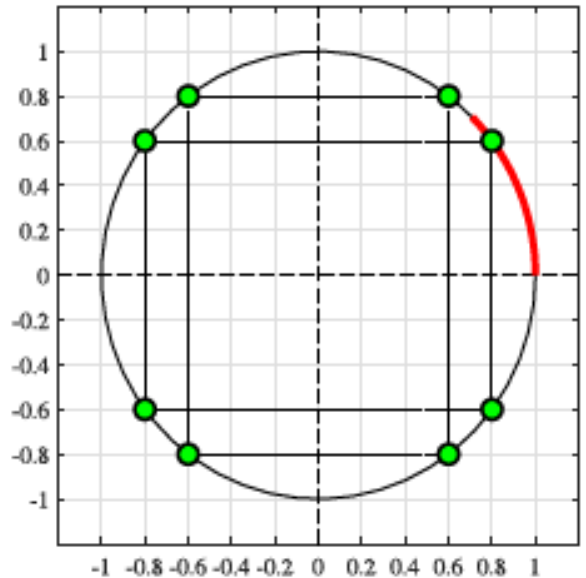

Fig. 1. The sparsity equivalence relation in $\mathrm{R}^{2}$. The green points represent the eight vectors of the class $[(4.3)]$ in the unit-sphere $\mathrm{S}^{2}$. The red thick arc represents the sparsity domain $\Omega_{2,2}$, which is the last, in the lexicographic order sense, half of the non-negative quadrant of $\mathrm{S}^{2}$.

Recall that, the equivalence relation $\sim_{s}$ partitions $\mathbb{R}^{n}$ into disjoints classes. Thus, any vector $\mathbf{v}$ belongs to a unique class $[\mathbf{w}]_{s}$ which will have a unique representative vector $\mathbf{w}$ in $\Omega_{\mathrm{g}}$. This is the reason behind the uniqueness of $\mathrm{w}$ in $\mathrm{H} 2 \mathrm{~s}$.

Then again, and according to $\mathrm{H} 1 \mathrm{~s}$, a vector $\mathbf{v}$ is sparsity equivalent to any other vector with the same (magnitudewise) components but rather these have been arbitrarily permuted, or have been all arbitrarily (but equally) scaled. Thus, without any loss in generality, each class could be represented by one of its vectors such that its components:

- have all positive signs, and

- have all been decreasingly ordered, and

- have all been normalized to sum up 1 .

In other words, we intersect the three sets, $\mathbb{R}_{+}^{n} \cap\left\{\mathbf{v}: v_{n} \leq\right.$ $\left.\cdots \leq v_{1}\right\} \cap\left\{\mathbf{v}:\|\mathbf{v}\|_{p}=1\right\}$, to define the sparsity domain $\Omega_{\mathrm{s}}$ in the next result.

Corollary 1 (Sparsity domain). From each class, we take its last, in the lexicographic order sense, non-negative element to define the sparsity domain

$$
\Omega_{s}(n, p)=\left\{\mathbf{w} \in \mathbb{R}_{+}^{n}:\|\mathbf{w}\|_{p}=1, w_{n} \leq \cdots \leq w_{1}\right\},
$$

where $\|\mathbf{w}\|_{p}=\left(\sum_{i=1}^{n}\left|w_{i}\right|^{p}\right)^{\frac{1}{p}}$ and $p>0$.

Proof. We only need to probe uniqueness. Assume $\exists \mathbf{w}, \mathbf{w}^{\prime} \in$ $[\mathbf{w}]_{s}$, different but both in $\Omega_{s}$. Then, $\left|w_{i}\right|=r\left|w_{i}^{\prime}\right|$, with $r>$ 0 , because $\mathbf{w} \sim_{s} \mathbf{w}^{\prime}$. But then, $1=\|\mathbf{w}\|_{p}=r\left\|\mathbf{w}^{\prime}\right\|_{p}=r$. Consequently $r=1$ and $\mathbf{w}=\mathbf{w}^{\prime}$ (i.e., they are the same). $\square$

That is, the sparsity domain $\Omega_{\mathrm{s}}$ gathers unique non-negative representative vectors from each sparsity equivalence class. Therefore, it will suffice to construct sparsity to operate on $\Omega_{\mathrm{s}}$. Furthermore, by construction, the sparsity domain $\Omega_{\mathrm{s}}$ is equal to the last, in the lexicographic order sense, $\frac{1}{n !}$ th fraction of the non-negative quadrant of the $n$-dimensional unit $p$-sphere $\mathbb{S}_{p}^{n}=\left\{\mathbf{w} \in \mathbb{R}^{n}:\|\mathbf{w}\|_{p}=1\right\}$. For example, in $\mathbb{R}^{2}$ and with $p=$ 2 , the sparsity domain is given by, $\Omega_{\mathrm{s}}(2,2)=\left\{\mathbf{w} \in \mathbb{R}_{+}^{2}\right.$ : $\left.\|\mathbf{w}\|_{2}=1, w_{2} \leq w_{1}\right\}$. This set is illustrated in Fig. 1, where it corresponds to the last, in the lexicographic order sense, half of the the non-negative quadrant of the unit sphere $\mathbb{S}^{2}$, where the vector $(0.8,0.6)$ uniquely represents the class $[(4,3)]_{s}$.
Importantly, the sparsity domain $\Omega_{\mathrm{s}}$ in (6) is a compact set and sparsity would potentially benefit from this property. Note also that the normalization, $\|\mathbf{w}\|_{p}=1$, is rather arbitrary and different values will also work due to the scale invariance aspect of $\mathrm{H} 1 \mathrm{~s}$. Moreover, the decreasing ordering of components, $w_{n} \leq \cdots \leq w_{1}$, will be handy to our proofs in the following Section 3.2 .

\section{Proposed Characterization}

The previous Section 2 introduced the framework, including the sparsity equivalence relation and sparsity domain. Under this framework, this Section introduces the axioms that will effectively characterize sparsity. Based on these axioms, fundamental properties of sparsity will be derived.

\subsection{Axioms of Sparsity}

According to the literature, the sparsity should follow Dalton's first law of inequality of wealth distribution [15]. We formalize this established result into our main axiom.

Axiom 1 (A1s Transfers). $\forall \mathbf{w} \in \Omega_{\mathrm{s}}$ and $c \in \mathbb{R}$ sufficiently small, with $0<\operatorname{sign}\left(w_{i}-w_{j}\right) c, \mathbf{d}_{i j}=\operatorname{sign}(c)\left(\mathbf{e}_{\mathbf{i}}-\mathbf{e}_{j}\right)$ :

$$
\frac{s\left(\mathbf{w}+c \mathbf{d}_{i j}\right)-s(\mathbf{w})}{c}>0 \text {, and is bounded, }
$$

where vector $\mathbf{e}_{i}$ is the ith canonical basis element of $\mathbb{R}^{n}$, and sign is the signum function.

In essence, axiom 1, denoted A1s for short in the sequel, states that, sparsity increases in the directions where relative differences between the components' magnitudes increase. Otherwise, sparsity will decrease.

Behind A1s we encounter Dalton's first law but written in terms of directional derivatives; thus A1s carries a differentiability requirement. Surprisingly, this is all the machinery we need to characterize sparsity, as long as the dimension is fixed asit commonly is. However, we will need an additional axiom if the dimension changes.

Axiom 2 (A2s Replication). $\forall w \in \Omega_{s}$ :

$$
s(\mathbf{w} \oplus \cdots \oplus \mathbf{w})=s(\mathbf{w}),
$$

where $\oplus$ concatenates vectors.

What A2s states is that, sparsity is dimension invariant. We are now ready to define sparsity.

Definition 1 (Sparsity). Sparsity is a real-valued measure function $s: \Omega_{\mathrm{s}} \rightarrow \mathbb{R}$ following $\mathrm{A} 1 \mathrm{~s}$. If the dimension changes, sparsity also follows A2s.

Traditionally, A1s has been derived from (various) inherent properties of sparsity [16], [17], [18], [19]. In this paper, instead, we demonstrate the sufficiency or opposite-direction path and derive a large set of fundamental properties via only A1s. To that end, we will show that, Definition 1 constitutes a minimal characterization of sparsity. In practice, this characterization provides a simplified test to qualify sparsity measures. 


\subsection{Fundamental Properties of Sparsity}

This Section demonstrates fundamental properties of sparsity, given by Definition 1, all of which are derived from A1s. For ease of understanding, all proofs utilize basic undergraduate-level multivariate calculus.

\section{Lemma 1 (P1 Continuity and differentiability). $\forall d \in \mathbb{R}^{n}$} unitary: $\lim _{c_{0} \rightarrow 0} s\left(\mathbf{w}+c_{0} \mathbf{d}\right)=s(\mathbf{w})$.

Proof. Note that for $n>2$ the vectors $\left\{\mathbf{d}_{i j}\right\} \subset \mathbb{R}^{n}$ can not form a basis of $\mathbb{R}^{n}$. Thus, we grow the dimension and include the vector $\mathbf{e}_{n+1}$ to the extended set $\left\{\tilde{\mathbf{d}}_{i j}\right\} \subset \mathbb{R}^{n+1}$, which now forms a basis of $\mathbb{R}^{n+1}$. Now, we can write $\tilde{\mathbf{d}}=$ $\mathbf{d} \oplus 0=\sum_{i, j} c_{i j} \tilde{\mathbf{d}}_{i j}+0 \mathbf{e}_{n+1}$ unitary, where the last (null) term keeps $\tilde{d}_{n+1}=0$ and affects all $\tilde{\mathbf{d}}_{i j}$ with $j=n+1$. Finally, by the Moore-Osgood theorem of iterated limits [20], $\lim _{\left\{q_{j}\right\} \rightarrow 0_{n}} s\left(\mathbf{w} \oplus 0+\sum_{i, j} c_{i j} \tilde{\mathbf{d}}_{i j}\right)=s(\mathbf{w} \oplus 0)$, with $c_{i j}=c c_{i j}^{\prime}$, where we have used that by A1s, $\lim _{c \rightarrow 0} s(\mathbf{w}+$ $\left.c \mathbf{d}_{i j}\right)=s(\mathbf{w})$. Thus, $s$ is continuous in all variables except on the $n+1$ th one (of which we can not affirm anything), i.e., $s$ is continuous in $\mathbb{R}^{n}$. A similar argument could be used to probe differentiability noticing that, by A1s, $\nabla_{\mathrm{d}_{i j}} s(\mathbf{w})<\infty$, where $\nabla_{\mathrm{d}_{i j}}$ is the directional derivative operator in the direction $\mathbf{d}_{i j}$.

Lemma 2 (P2 Monotonicity). $\forall \mathbf{w}, \tilde{\mathbf{w}}$, which are equal except in two components, $\mathbf{w}=\left(w_{1}, \ldots, w, \ldots, 1-w, \ldots, w_{n}\right)$, and $\tilde{\mathbf{w}}=\left(w_{1}, \ldots, \tilde{w}, \ldots, 1-\tilde{w}, \ldots, w_{n}\right)$, with $\frac{1}{2} \leq w<\tilde{w} \leq 1$ : $s(\mathbf{w})<s(\tilde{\mathbf{w}})$.

Proof. Let the indices of these different components be $i$ and $j$. First, by $\frac{1}{2} \leq w<\tilde{w}$, we have that $1-w \leq w$ and $1-\tilde{w}<\tilde{w}$. Then, $\tilde{\mathbf{w}}$ is constructed from $\mathbf{w}$ by moving $c=$ $\tilde{w}-w>0$ from $w_{j}=1-w$ to $w_{i}=w$, i.e., $\tilde{\mathbf{w}}=\mathbf{w}+c \mathbf{d}_{i j}$ with $\mathbf{d}_{i j}=\mathbf{e}_{i}-\mathbf{e}_{j}$. Now, by A1s, we have that $s(\tilde{\mathbf{w}})>$ $s(\mathbf{w})$. However, we need to lastly verify that $c \leq 1-w$ (maximum possible mass to reallocate). To do this, note that $w<\tilde{w} \leq 1$ thus $0<c=\tilde{w}-w \leq 1-w$.

Lemma 3 (P3 Surjectivity). $\forall c_{1} \in\left(\inf s\left(\Omega_{\mathrm{s}}\right), \sup s\left(\Omega_{\mathrm{s}}\right)\right), \exists \mathbf{w}$ : $s(\mathbf{w})=c_{1}$.

Proof. It follows from P1 (thus A1s) and P2 (also A1s).

Lemma 4 (P4 Extremes). $\forall \mathbf{w} \nsim_{s}\left\{\mathbf{e}_{1}, \mathbf{1}_{n}\right\}: s\left(\mathbf{1}_{n}\right)<s(\mathbf{w})<$ $s\left(\mathbf{e}_{1}\right)$. Moreover, $s\left(\mathbf{1}_{n}\right)<s\left(\mathbf{1}_{n-1} \oplus 0\right)<\cdots<s\left(\mathbf{e}_{1}\right)$.

Proof. We will probe that $s(\mathbf{w})<s\left(\mathbf{e}_{1}\right)$ via $n-1$ concentration steps. To add in generality, assume the components of $\mathbf{w}$ be randomly permuted. First, set $\mathbf{w}^{(0)}=\mathbf{w}$ and find $i=$ $\arg \min _{k} w_{k}-\|\mathbf{w}\|_{\infty}$. Now, for $t=1, \ldots, n-1$, find index $j=\arg \min _{k} w_{k}^{(t-1)}-\left\|\mathbf{w}^{(t-1)}\right\|_{-\infty}$, and construct

$$
\mathbf{w}^{(t)}=\left\{\begin{array}{l}
w_{i}^{(t)}=w_{i}^{(t-1)}+w_{j}^{(t-1)} \\
w_{j}^{(t)}=0 \\
w_{k}^{(t)}=w_{k}^{(t-1)}, k \neq i, j,
\end{array}\right.
$$

i.e., at each iteration $t$, we do $\mathbf{w}^{(t)}=\mathbf{w}^{(t-1)}+c \mathbf{d}_{i j}$ with $c=$ $w_{j}^{(t-1)}>0$ and $\mathbf{d}_{i j}=\mathbf{e}_{i}-\mathbf{e}_{j}$. Hence, by A1s, $s\left(\mathbf{w}^{(t)}\right)>$ $s\left(\mathbf{w}^{(t-1)}\right)$. Finally, note that in the last iteration, all the mass is concentrated in one single coefficient, $\mathbf{w}^{(n-1)} \sim_{s} \mathbf{e}_{1}$.

A similar proof holds for $s\left(\mathbf{1}_{n}\right)<s(\mathbf{w})$.
Lemma 5 (P5 Semistrict quasiconvexity). $\forall \mathbf{w}, \tilde{\mathbf{w}}$ with $s(\mathbf{w}) \neq s(\tilde{\mathbf{w}})$, and $\forall c_{2} \in(0,1): s\left(c_{2} \mathbf{w}+\left(1-c_{2}\right) \tilde{\mathbf{w}}\right)<\max$ $\{s(\mathbf{w}), s(\tilde{\mathbf{w}})\}$.

Proof. By the scale invariance in $\mathrm{H} 2 \mathrm{~s}$, write sparsity as the ratio $s=f$, with functions $f, g$ homogeneous, symmetric and sign invariant. Furthermore, let $f, g$ be non-negative, differentiable, $g$ non-null, and such that $f(\mathbf{w})<f(\tilde{\mathbf{w}})$ and $g(\mathbf{w})>g(\tilde{\mathbf{w}})$. Then, $s(\mathbf{w})<s(\tilde{\mathbf{w}})$ and, by A1s, we can assume $\tilde{\mathbf{w}}=\mathbf{w}+c \mathbf{d}_{i j}$ with $c>0$. (We have that $\tilde{\mathbf{w}} \nsim_{s} \mathbf{w}$ with $s(\mathbf{w}) \neq s(\tilde{\mathbf{w}})$.) Now, by the differentiability of $f$, $\frac{f(\tilde{w})-f(\mathbf{w})}{c} \approx \nabla_{\mathbf{d}_{i j}} f(\mathbf{w})=\frac{\partial f}{\partial w_{i}}(\mathbf{w})-\frac{\partial f}{\partial n j}(\mathbf{w})>0$, where the last inequality is due to $f(\mathbf{w})<f(\tilde{\mathbf{w}})$. By a simple continuity argument, one may relax the differentiability to a continuity requirement. Now, by the symmetry of $f$, and writing $\mathbf{w}=w_{i} \oplus w_{j} \oplus \mathbf{w}^{k}, \quad \frac{\partial f}{\partial w_{i}}\left(w_{i} \oplus w_{j} \oplus \mathbf{w}^{k}\right)>\frac{\partial f}{\partial w_{i}}\left(w_{j}\right.$

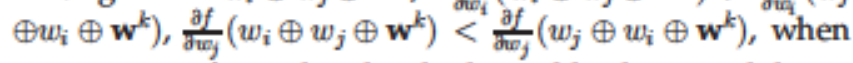
$w_{i}>w_{j}$, i.e., for each individual variable, the partial derivative of $f$ grows accordingly to the value of that variable. Hence, $f$ is convex in each variable thus convex in all of them. Similarly, $g$ is concave. Finally, use [13, Theorem 2.3.8] which proves the ratio $s=\frac{f}{g}$ is semistrict quasiconvex.

Lemma 6 (P6 Schur convexity). $\forall \mathbf{w} \succ \tilde{\mathbf{w}}: s(\mathbf{w})>s(\tilde{\mathbf{w}})$, where $\succ$ denotes "majorizes (dominates)".

Proof. It follows from H2s (symmetry part) and P5 (by A1s), c.f. [21, Prop. 11].

Lemma 7 (P7 Regularity). $\forall \mathbf{w}$ with $w_{1} \geq w_{j}, \forall j \neq 1$, and $0 \leq c_{3}<c_{4}: s\left(\mathbf{w}+c_{3} \mathbf{e}_{1}\right)<s\left(\mathbf{w}+c_{4} \mathbf{e}_{1}\right)$.

Proof. To add in generality, let the components of $\mathbf{w}$ be randomly permuted. First, find $i=\arg \min _{k} w_{k}-\|\mathbf{w}\|_{\infty}$, define $\tilde{\mathbf{w}}=c_{6}\left(\mathbf{w}+c_{3} \mathbf{e}_{i}\right)$, with $c_{3}>0, c_{6}=1+c_{7}, c_{7}<1$, and set $\tilde{\mathbf{w}}^{(0)}=\tilde{\mathbf{w}}$. Now, for $t=1, \ldots, n-1$, find index $j$ such that, $j=\arg \min _{k} w_{k}^{(t-1)}-\left\|\mathbf{w}^{(t-1)}\right\|_{-\infty}$, and construct

$$
\tilde{\mathbf{w}}^{(t)}=\left\{\begin{array}{l}
\tilde{w}_{i}^{(t)}=\tilde{w}_{i}^{(t-1)}+c_{7} w_{j} \\
\tilde{w}_{j}^{(t)}=\tilde{w}_{j}^{(t-1)}-c_{7} w_{j}=w_{j} \\
\tilde{w}_{k}^{(t)}=\tilde{w}_{k}^{(t-1)}, k \neq i, j,
\end{array}\right.
$$

i.e., at each iteration $t$, we do $\tilde{\mathbf{w}}^{(t)}=\tilde{\mathbf{w}}^{(t-1)}+c \mathbf{d}_{i j}$, with $c=c_{7} w_{j}>0$ and $\mathbf{d}_{i j}=\mathbf{e}_{i}-\mathbf{e}_{j}$. Hence, by A1s, $s\left(\tilde{\mathbf{w}}^{(t)}\right)>$ $s\left(\tilde{\mathbf{w}}^{(t-1)}\right)$. Finally, in the last iteration, $\tilde{\mathbf{w}}^{(n-1)}=\mathbf{w}+c_{4} \mathrm{e}_{\mathrm{i}}$, with $c_{4}=c_{3}+c_{7}\left(c_{3}+\sum_{k} w_{k}\right)>c_{3}$.

Lemma 8 (P8 Homogeneous growth). $\forall w \varkappa_{s} 1_{n}$, and $\forall c_{5}>$ $0: s\left(\mathbf{w}+c_{5} \mathbf{1}_{n}\right)<s(\mathbf{w})$.

Proof. First, by H2s, $\mathbf{w}+c_{5} \mathbf{1}_{n} \sim_{s} c_{8} \mathbf{w}+\left(1-c_{8}\right) \mathbf{1}_{n}$, with $c_{8}=$ $\frac{1}{1+c_{5}}<1$. Now, by P5, $s\left(\mathbf{w}+c_{5} \mathbf{1}_{n}\right)<\max \left\{s(\mathbf{w}), s\left(\mathbf{1}_{n}\right)\right\}$. Finally, by P4 (by A1s), $\max \left\{s(\mathbf{w}), s\left(\mathbf{1}_{n}\right)\right\}=s(\mathbf{w})$.

Lemma 9 (P9 Compositions). $\forall \mathbf{w}, \tilde{\mathbf{w}}$ with $s(\mathbf{w})<s(\tilde{\mathbf{w}})$, and any increasing transformation $\Theta: s\left(\Omega_{s}\right) \rightarrow \mathbb{R}$ (with operation domain $\left.s\left(\Omega_{\mathrm{s}}\right)\right): \Theta \circ s(\mathbf{w})<\Theta \circ s(\tilde{\mathbf{w}})$, where $\circ$ composes functions.

Proof. Trivial, cf. [21, Prop. 9].

Moreover, when the dimension changes, sparsity, given by Definition 1, satisfies the next additional properties.

Lemma 10 (P10 Completeness). $\forall \mathbf{w}: s(\mathbf{w} \oplus 0)>s(\mathbf{w})$. 
Proof. (From [4].) First, by A2s, $s(\mathbf{w})=s(\mathbf{w} \oplus \cdots \oplus \mathbf{w})$ $(n+1$ replicas of $\mathbf{w})$. Then, by $\mathbf{A} 1 \mathrm{~s}$

$$
s(\mathbf{w} \oplus \cdots \oplus \mathbf{w})<s\left(\frac{n+1}{n}(\mathbf{w} \oplus \cdots \oplus \mathbf{w}) \oplus \mathbf{0}_{n}\right) .
$$

Now, by $\mathrm{H} 2 \mathrm{~s}$ (scale invariance part), the right hand side of (11) equals $s\left(\mathbf{w} \oplus \cdots \oplus \mathbf{w} \oplus \mathbf{0}_{n}\right)$. Finally, again, by A2s, $s\left(\mathbf{w} \oplus \cdots \oplus \mathbf{w} \oplus \mathbf{0}_{n}\right)=s(\mathbf{w} \oplus 0)$.

Lemma 11 (P11 Maxima). $\forall n \in \mathbb{Z}_{+}: s\left(\mathbf{1}_{n} \oplus 0\right)>s\left(\mathbf{1}_{n}\right)=$ $s\left(\mathbf{1}_{n+1}\right)$.

Proof. The inequality follows from P10 (by A1s), with $\mathbf{w}=$ $\mathbf{1}_{n}$. And, the equality follows from A2s, with $\mathbf{w}=1$.

These Lemmas 1-11 are not limited to $\Omega_{\mathrm{s}}$ of non-negative vectors. In fact, the Lemmas hold on the entire set $\mathbb{R}^{n}$ of real vectors via the sparsity equivalence. In brief, the Lemmas demonstrate that sparsity has the following properties:

- continuous and differentiable (P1),

- monotone (P2),

- surjective (P3),

- bounded (P4),

- semistrict quasiconvex (P5), and

- Schur convex (P6).

Actually, because sparsity is continuous and the sparsity domain is a compact set, we could probe that the extreme value and mean value theorems in several variables hold, which together imply properties P2-P4. Importantly, the discovered semistrict quasiconvexity nature of sparsity will be essential to construct new measures and go beyond traditional convex sparsity.

\subsection{Axiomatic Characterization of Entropy}

To this point, we have addressed sparsity exclusively, since its counter-part, entropy, denoted $h$ in the sequel, could be analogously defined as the arithmetic or geometric inverse of sparsity. Indeed, in our framework, sparsity and entropy are complementary measures, c.f. [8], [22]. That is, sparsity measures the simplicity or compressibility of a signal $\mathbf{x} \in$ $\mathbb{R}^{n}$ in a possibly invertible transformation domain, e.g., wavelets. Hence, sparsity is applied to a set of coefficients $\mathbf{w}$ that carry information about $\mathbf{x}$. Analogously, entropy measures the complexity or uncertainty of $\mathbf{x}$ in the non-invertible outcome frequency domain, e.g., $b$-bins normalized histogram. Thus, entropy is applied to an empirical probability distribution p. We adapt then the following hypotheses, axioms and definition to entropy.

Hypothesis 3 ( $\mathrm{H} 1 \mathrm{~h}$ Entropy equivalence). For any pair of vectors $\mathbf{p}, \mathbf{q} \in[0,1]^{b}$, we say that "q is entropy equivalent to $\mathbf{p}^{\prime \prime}$, denoted $\mathbf{q} \sim_{\mathrm{s}} \mathbf{p}$, and meaning, both $\mathbf{q}$ and $\mathbf{p}$ have the same level of entropy, if one vector is a permutation of the other, $\mathbf{q} \sim_{\mathbf{s}} \mathbf{p} \equiv \mathbf{q}=\pi(\mathbf{p})$.

Hypothesis 4 (H2h Entropy assessment). $\forall q \in[0,1]^{b}$, $\exists ! \mathbf{p} \in \Omega_{\mathrm{h}}$ (entropy domain): $\mathbf{q} \sim_{\mathbf{s}} \mathbf{p} \quad \Rightarrow \quad h(\mathbf{q})=h(\mathbf{p})$.

Note that we are using the sparsity equivalence $\sim_{s}$ in $\mathrm{H} 1 \mathrm{~h}-\mathrm{H} 2 \mathrm{~h}$. However, since $\mathrm{p}$ is positive (it contains relative frequencies, i.e., $\left.p_{i} \in[0,1]\right)$, it is enough to consider the permutation of components of $\mathbf{p}$ in both hypotheses. Similarly, the entropy domain is given by the following result.
Corollary 2 (Entropy domain). From each class we take its last, in the lexicographic order sense, element to define the entropy domain, $\Omega_{\mathrm{h}}(b, 1)=\left\{\mathbf{p} \in[0,1]^{b}:\|\mathbf{p}\|_{1}=1, p_{b} \leq \cdots \leq p_{1}\right\}$.

As in the sparsity domain (6), the normalization $\|\mathbf{p}\|_{1}=1$ is rather arbitrary. That is, it is possible to consider non-normalized histograms $\mathbf{p} \in \mathbb{Z}_{+}^{b}$, e.g., $\|\mathbf{p}\|_{1}=n$ with $n$ is the number of elements of $\mathbf{x}$. Moreover, the normalization could be based on a different $p$-norm, but the natural one is $p=1$.

Overall, the complementary behavior entropy is postulated in the inequality-inverted versions of A1s and A2s.

Axiom 3 (A1h Transfers). $\forall \mathbf{p} \in \Omega_{\mathrm{h}}$ and $c \in \mathbb{R}$ sufficiently small, with $0<\operatorname{sign}\left(p_{i}-p_{j}\right) c, \mathbf{d}_{i j}=\operatorname{sign}(c)\left(\mathbf{e}_{i}-\mathbf{e}_{j}\right)$ :

$$
\frac{h\left(\mathbf{p}+c \mathbf{d}_{i j}\right)-h(\mathbf{p})}{c}<0 \text {, and is bounded, }
$$

where this time vector $\mathbf{e}_{i}$ is the ith canonical basis element of $\mathbb{R}^{b}$.

Axiom 4 (A2h Replication). $\forall \mathbf{p} \in \Omega_{\mathrm{h}}: h(\mathbf{p} \oplus \cdots \oplus \mathbf{p})>$ $h(\mathbf{p})$.

Which together, A1h and A2h, define entropy.

Definition 2 (Entropy). Entropy is a real-valued measure function $h: \Omega_{\mathrm{h}} \rightarrow \mathbb{R}$ following A1 $\mathrm{h}$. If the dimension changes, entropy also follows $\mathrm{A} 2 \mathrm{~h}$.

As a consequence of the inverted inequalities in A1h, entropy will follow the fundamental properties of sparsity but with inverted inequal ities in Lemmas 2, 4, 5, 6, 7, 8, and 9. Moreover, due to A2h, it will follow $h(\mathbf{p} \oplus 0)=h(\mathbf{p})$ and $h\left(\mathbf{1}_{b} \oplus 0\right)=$ $h\left(\mathbf{1}_{b}\right)<h\left(\mathbf{1}_{b+1}\right)$ in Lemmas 10 and 11, respectively. In practice, entropy ignores zeros and sparsity considers them.

Next we will construct concrete instances of sparsity and entropy following the findings from this Section.

\section{Proposed Measures}

The main contribution of Section 3 was the minimal characterization and fundamental properties of sparsity. In this Section, we give a step forward on the systematic construction of measures of sparsity.

\subsection{Functional Form}

The comprehensive analysis in [23] shows that generalized sparsity follows a quotient-of-functions form. This, together with the discovered semistrict quasiconvexity of sparsity, Lemma 5, motivates the following theorem.

Theorem 3 (Functional form). Let the functions $f, g$ be continuous, homogeneous, sign invariant, and symmetric. If the function

$$
s(\mathbf{w})=\frac{f(\mathbf{w})}{g(\mathbf{w})},
$$

measures sparsity, then

- $f$ is positive convex, and $g$ is positive concave

- $f$ is negative convex, and $g$ is positive convex

- $f$ is convex, and $g$ is positive affine.

Proof. It follows from the proof of Lemma 5. Other configurations of $f$ and $g$ (with $f$ negative convex and $g$ positive convex, or $f$ convex and $g$ positive affine) work as well (c.f. [13, Theorem 2.3.8]). 


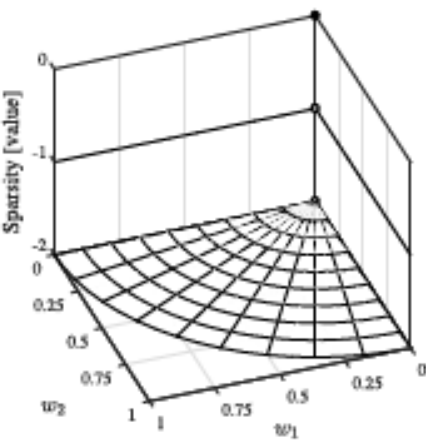

(a) $-\|\mathbf{w}\|_{0}$

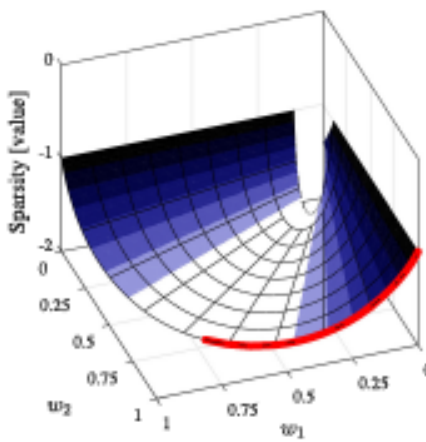

(b) $s_{\text {eff }}\left(w ; 1,1^{+}\right)$

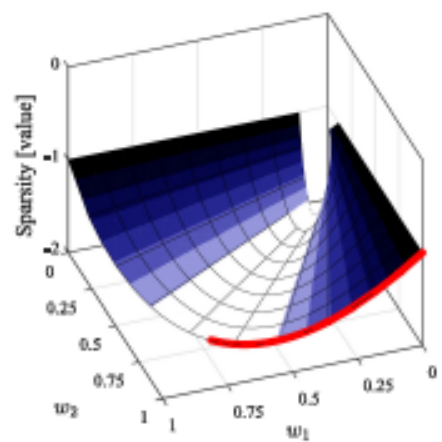

(c) $s_{\text {eff }}(\mathbf{w} ; 1,2)$

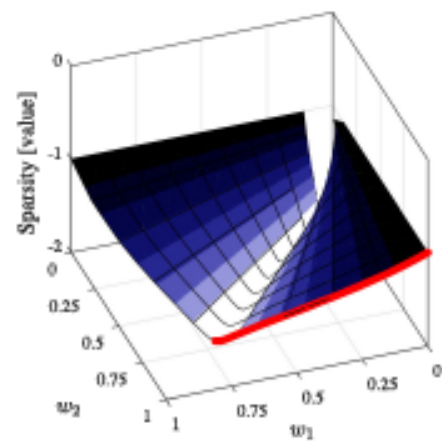

(d) $s_{\text {eff }}(\mathbf{w} ; 1,+\infty)$

Fig. 2. Sparsity in the non-negative quadrant of the $\mathrm{S}^{2}$ unit-sphere. Measures include the canonical strict sparsity $-\|\mathbf{w}\|_{0}$ (Fig. 2a), and effective sparsity (Figs. 2b-2d). For ease of comparison, values are normalized to $[0,2]$. The thick red curve represents the sparsity values at the sparsity domain $\Omega_{2,2,1}$, with minimum at point $(\sqrt{2}, \sqrt{2}) \in\left[\mathbf{1}_{2}\right]_{\mathrm{s}}$ and maximum at $(0,1) \in\left[\mathbf{e}_{1}\right]_{\mathrm{s}}$. Note that effective sparsity is constant along the same radial directions, according to H2s. Similar shapes would be drawn by $-h_{\text {etf }}(\mathbf{w} ; \alpha, \beta)$.

Importantly, the functional form (13) outlines the basic structure of sparsity measures. Based then on the requirements of Theorem 3 for functions $f$ and $g$, we could design specific measures of sparsity as follows.

Corollary 4 (Effective sparsity). The function

$$
s_{\mathrm{eff}}(\mathbf{w} ; \alpha, \beta)=\frac{\langle\mathbf{w}\rangle_{\beta}}{\langle\mathbf{w}\rangle_{\alpha}}, \quad \alpha \leq 1 \leq \beta, \alpha \neq \beta,
$$

defined on $\Omega_{\mathrm{s}}$, measures sparsity. In (14), the function $\langle.\rangle_{p}=$ $\operatorname{Card}(.)^{-\frac{1}{p}}\|\cdot\|_{p}$ is the generalized or power p-mean [24], and Card computes the cardinality.

Proof. Weneed to provethat H1s, A1s and A2s hold. Clearly, $\mathrm{H} 1 \mathrm{~s}$ holds. It is also clear that $s_{\text {eff }}$ is continuous (composition of continuous functions), then P1 holds. And, by the Cauchy-Schwarz inequality, P4 holds. Then, by [19], P1, P4 and decomposability (a property to be verified later) imply A1s. Finally, the factor $n^{\alpha}$ makes A2s to hold.

Intuitively, if vector $\mathbf{w}$ has $k=\|\mathbf{w}\|_{0}$ non-zero components, by the Cauchy-Schwarz inequality, we have that

$$
s_{\text {eff }}(\mathbf{w} ; \alpha, \beta) \geq\left(\frac{k}{n}\right)^{\frac{1}{\beta} \frac{1}{\alpha}},
$$

i.e., when $k$ decreases or as the signal becomes more sparse, effective sparsity increases. Actually, the effective sparsity resembles the canonical strict sparsity [25] $-\|\mathbf{w}\|_{0}: \mathbb{R}_{+}^{n} \rightarrow \mathbb{Z}_{-}$, exœpt at the origin $0_{n}$, where $s_{\text {eff }}$ is clearly not defined, $\lim _{c_{6} \rightarrow 0} s_{\text {eff }}\left(c_{6} \mathbf{1}_{n} ; \alpha, \beta\right) \neq \lim _{c_{6} \rightarrow 0} s_{\text {eff }}\left(c_{6} \mathbf{e}_{1} ; \boldsymbol{\alpha}, \beta\right)$. Fig. 2 illustrates this resemblance. In general, pammeters $\alpha$ and $\beta$ will tune the sensitiveness to sparseness of effective sparsity. For instanc, with $\alpha=1$ fixed and $\beta \rightarrow 1^{+}$, very sparse signals are better discriminated; but as $\beta \rightarrow+\infty$ effective sparsity become less selective. Graphically, this could be observed in the different curvatures of the shapes drawn by the sparsity values at points in the sparsity domain $\Omega_{\mathrm{S}}$, represented by a thick red arc in Fig. 2 .

Analogously, for entropy we have the next measure.

\section{Corollary 5 (Effective entropy). The function}

$$
h_{\mathrm{eff}}(\mathbf{p} ; \alpha, \beta)=\frac{\|\mathbf{p}\|_{\alpha}}{\|\mathbf{p}\|_{\beta}}, \quad \alpha \leq 1 \leq \beta, \alpha \neq \beta,
$$

defined on $\Omega_{\mathrm{h}}$, measures entropy.
Proof. Apply the geometric inverse $\frac{1}{x}$ to $s_{\text {eff }}$, but remove the dimension-dependency scaling factor.

\subsection{Operational Meaning}

The fundamental properties of sparsity, Lemmas 1-9, provide valuable geometrical information of sparsity. However, these properties do not reveal its operational meaning. This interpretation is encoded in the convexity of functions $f$ and $g$ in Theorem 3, and the complete symmetry of $\mathbb{R}^{n}$ under $\mathrm{H} 1 \mathrm{~s}$, which is embedded in the sparsity domain $\Omega_{s}$. Concretely, the difference in convexity between $f$ and $g$ expresses how distant a vector $\mathbf{w}$ is from the class $\left[\mathbf{1}_{n}\right]$ which establishes the sparsity lower bound. Fundamentally, the effective sparsity of a vector $\mathbf{w}$ is its geodesic distance to the class $\left[\mathbf{1}_{n}\right]$. We have proven the following corollary.

Corollary 6 (Operational-geometrical meaning). The effective sparsity of vector $\mathbf{w}$ is its geodesic distance to the nearest dement in the class $\left[\mathbf{1}_{n}\right]$ on a surface $\mathcal{S}$ sparsity equivalent to $\Omega_{\mathrm{s}}$

$$
\Theta_{\mathcal{S}} \circ s_{\mathrm{eff}}(\mathbf{w} ; \alpha, \beta)=\underset{\mathcal{S} \sim \Omega_{s}}{\operatorname{Geodesic}}\left(\mathbf{w},\left[\mathbf{1}_{n}\right]\right),
$$

where function $\Theta_{\mathcal{S}}$ depends on $\mathcal{S}$ and is increasing, c.f. Lemma 9 .

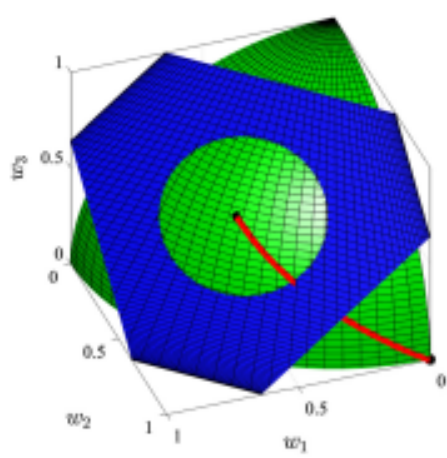

Fig. 3. The operational meaning of effective sparsity in $\mathbf{R}^{3}$. The unitradius $\beta$-ball (with $\beta=2$ ), represented by the green surface in the "back", intersects the $\mathrm{kn}^{\frac{1}{\beta} \frac{1}{\alpha}}$-radius $\alpha$-ball (with $\alpha=1$ ), represerted by the blue surface in the "front". The effective sparsity of a vector $\mathbf{v}$ is its geodesic distance to the class $\left[\mathbf{1}_{\mathrm{n}}\right]$, i.e., the minimum distance (on a surface $\mathcal{S}$ ) to the closest vector $\mathbf{w} \sim \mathbf{1}_{\mathrm{n}}$. The geodesic between the classes $\left[\mathbf{1}_{\mathrm{n}}\right]$ and $\left[\mathbf{e}_{1}\right]$ is represented by a thick red arc. 


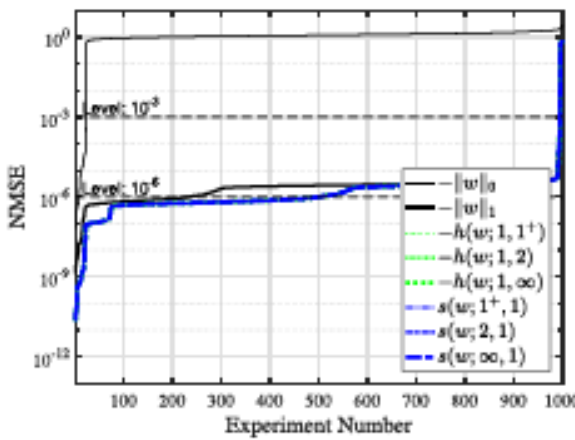

(a) Expected spikes $10 \%$, samples $40 \%$

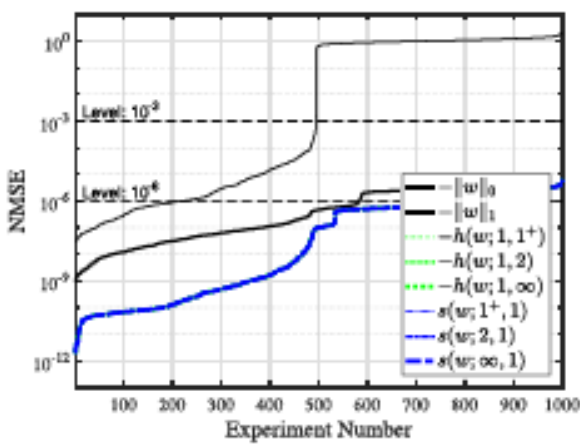

(b) Expected spikes $10 \%$, samples $50 \%$

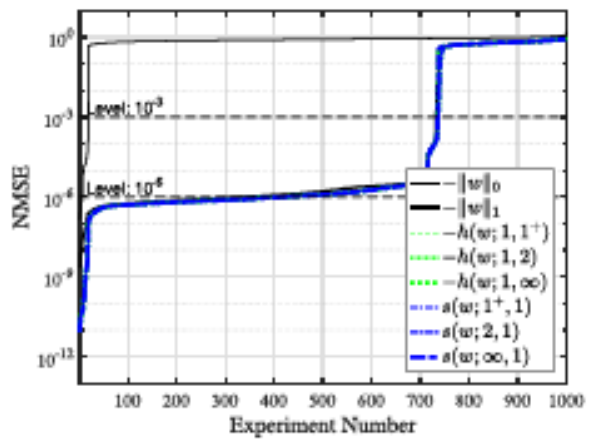

(d) Expected spikes $20 \%$, samples $40 \%$

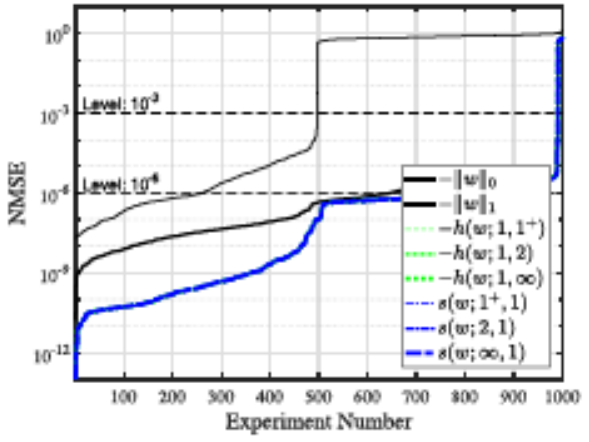

(e) Expected spikes $20 \%$, samples $50 \%$

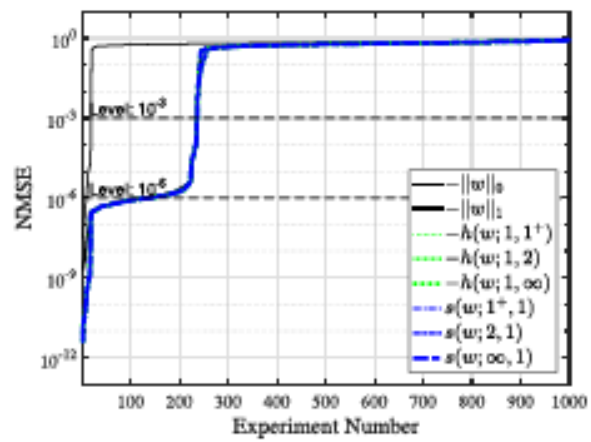

(g) Expected spikes $30 \%$, samples $40 \%$

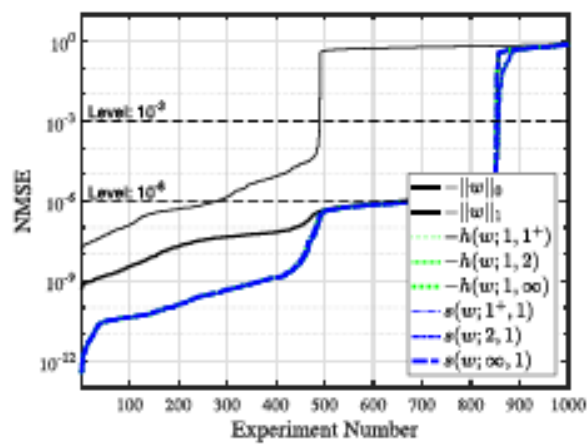

(h) Expected spikes $30 \%$, samples $50 \%$

Fig. 4. Normalized mean squared error (NMSE) (sorted values) of the sparse recovery of 1000-dimensional binary signals, with variable percentage of expected 1's (columns) and of samples (rows) (both in percentage scale). The effective sparsity and $-\|$. $\|_{1}$ achieve fundamentally the same solution. Nevertheless, effective sparsity achieve slightly lower error levels.

Fig. 3 illustrates this geometrical interpretation of effective sparsity. All points in the intersection of an $\alpha$-ball and a $\beta$-ball have the same sparsity, which can be measured in the geodesic between classes $\left[\mathbf{1}_{n}\right]$ and $\left[\mathbf{e}_{1}\right]$ (the sparsity upper bound), represented by a thick red arc. For instance, if parameters $\alpha=1$ and $\beta=2$, and surface $\mathcal{S}$ is the unit 1-ball or unit 2-ball then

$$
\begin{aligned}
\left(\frac{1}{n} s_{\text {eff }}(\mathbf{w} ; 1,2)^{2}-1\right)^{\frac{1}{2}} & =\underset{\|\mathbf{w}\|_{1}=1}{\operatorname{Gesic}}\left(\mathbf{w},\left[\mathbf{1}_{n}\right]\right), \\
\sec ^{-1}\left(s_{\mathrm{eff}}(\mathbf{w} ; 1,2)\right) & =\underset{\|\mathbf{w}\|_{2}=1}{\operatorname{Gedesic}}\left(\mathbf{w},\left[\mathbf{1}_{n}\right]\right),
\end{aligned}
$$

respectively. Note that the above expressions constitute alternative paths to compute sparsity and geodesics.

This Section has demonstrated the functional quotient-offunctions form of sparsity. From this functional form, we have constructed and interpreted effective sparsity. Next, in the coming Section, both effective sparsity and entropy will be numerically evaluated.

\section{Performance Evaluation}

Sparsity is the key notion for sparse recovery. As such, we will evaluate the recovery accuracy of effective sparsity. Its computation time however will not be assessed because slower resolution times are expected from effective sparsity due to its non-convexity [1]. Nevertheless, we will evaluate the computation time of effective entropy in signals' complexity analysis, whose traditional tools are computationally-demanding algorithms [26]. Its accuracy will not be assessed though because entropy formulations possess different rationale thus scale; although, all of them should respect the same entropy-based ordering [27]. Therefore, it is our goal to qualify effective sparsity and effective entropy via standard benchmarks. All evaluations are completed using MATLAB 9.6 (R2019a) in a INTEL ${ }^{\star} \mathrm{CORE}^{\mathrm{TM}} \mathrm{i} 7-6820 \mathrm{HQ}$ processor (8M Cache, $2.70 \mathrm{GHz}$ ).

\subsection{Recovery Accuracy of Effective Sparsity}

In Compressed Sensing [1], a signal $\mathbf{x} \in \mathbb{R}^{n}$ is recovered from samples $\mathbf{y} \in \mathbb{R}^{m}$, with $m \ll n$, under the assumption that $\mathbf{x}$ possesses a sparse representation $\mathbf{w} \in \mathbb{R}^{n}$ in a given domain. Formally, this reads

$$
\mathbf{w}^{\#}=\arg \max _{\mathbf{w}}\{s(\mathbf{w}): \Phi \Psi \mathbf{w}=\mathbf{y}\},
$$

where $\Phi \in \mathbb{R}^{m \times n}$ and $\Psi \in \mathbb{R}^{n \times n}$ are the sensing and sparsifying matrices, respectively, and ." denotes "recovered". Then, the original signal is reconstructed from $x^{\#}=\Psi w^{\#}$.

Recently, non-convex measures have been proposed to promote sparsity with the aim to reduce the number of samples in (20) [2]. Among these proposals, effective sparsity appeared in the form of the ratio $\|\mathbf{w}\|_{1} /\|\mathbf{w}\|_{\infty}$ to induce the sparsity of probability measures within a convex formulation of (20) [3] (also [28]). However, a recurrent instance of effective sparsity is the ratio $\|\mathbf{w}\|_{1} /\|\mathbf{w}\|_{2}$ [5], which provided geometric meaning [14] and recovery guarantees [29]. Therefore, we will solve (20) using the two aforementioned ratios, and the ratio with one parameter set to 1 and the other approaching 1 (from below or above) which corresponds to the Shannon entropy solution [9], [10]. The benchmark will be against classical approaches, i.e., maximizing $-\|\cdot\|_{0}$ and its proxy $-\|\cdot\|_{1}$. 


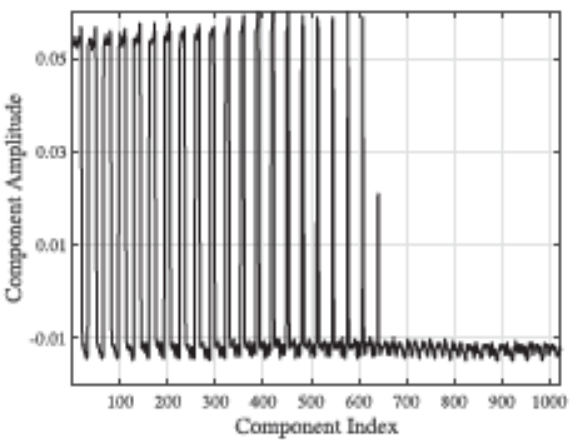

(a) Original w (TV coefficients)

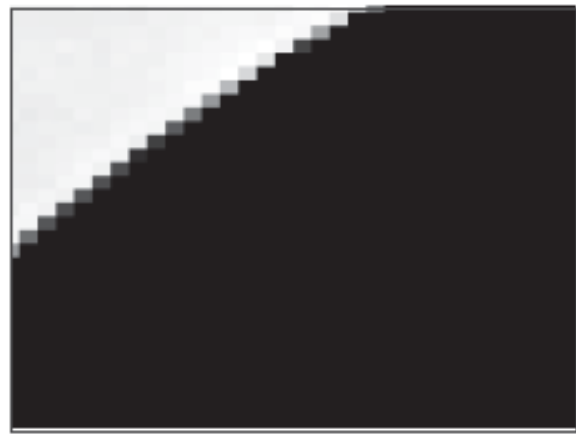

(d) Original $\mathbf{x}$ (an extract)

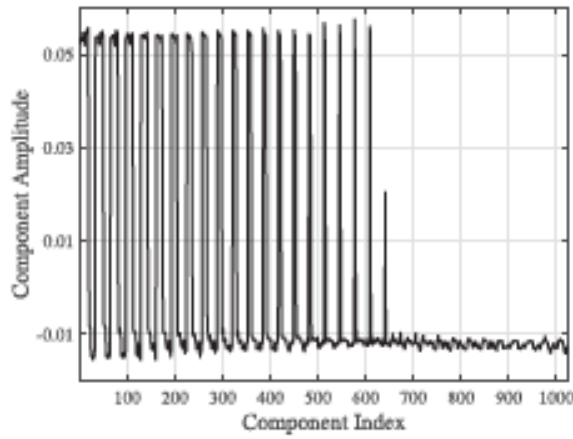

(b) Recovered w\#, $-\|\cdot\|_{1}$ solution

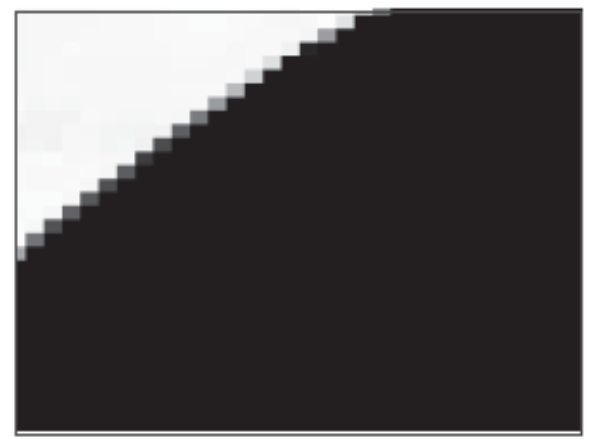

(e) Recovered $\mathbf{x}^{\#},-\|\cdot\|_{1}$ solution

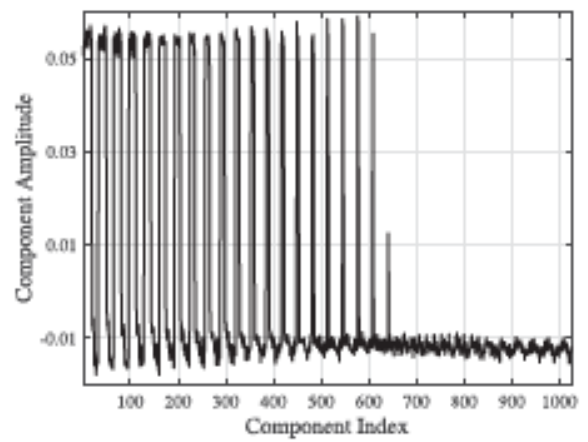

(c) Recovered $w^{\#},\|\cdot\|_{1} /\|\cdot\|_{2}$ solution

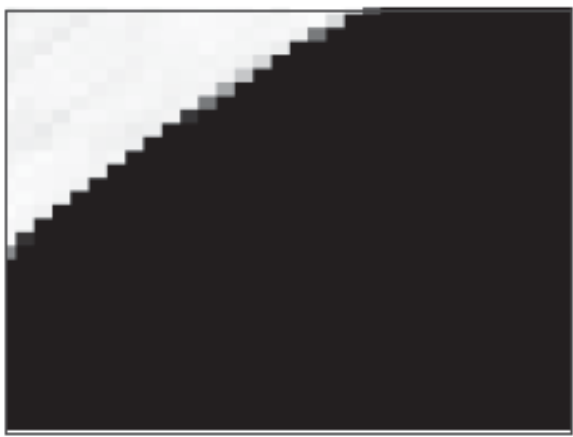

(f) Recovered $\mathbf{x}^{\#},\|\cdot\|_{1} /\|,\|_{2}$ solution

Fig. 5. An extract of the TV-based recovery of the classical cameraman benchmark. (Top) TV coefficients of the original and recovered solutions via the convex proxy $-\|\cdot\|_{1}$ and effective sparsity $\|\cdot\|_{1} /\|\cdot\|_{2}$. (Bottom) Recovered images corresponding to the above TV coefficients.

A generic interior-point method-based solver with identical configuration parameters is employed to get all solutions. However, because of this generic aspect of the solver, an additional verification step is needed for effective sparsity: the corrected solution is the sparsest solution (measured via the effective sparsity itself) among the set of all computed solutions. This verification step would help to reduce the number of local solutions for effective sparsity. Finally, the recovery accuracy is assessed using the normalized mean squared error, NMSE $=\frac{1}{n} \frac{\|w\|-w \|_{2}}{\|w\|_{2}}$.

Two type of signals will be studied following the common practice in compressed sensing. The first type corresponds to a partly-artificial signal model with few non-zero components, which actually appears in real systems, e.g., in binary signals decoding [1]. The second type follows a more realistic signal model which incorporates power-law decaying components representing noise from measurement or sparsification inaccuracies [1].

\subsubsection{Sparse Signals, e.g., Decoding Problems}

The signal $\mathrm{x}$ has components $x_{i}$ which take on the value 1 with probability $\kappa$

$$
x_{i} \sim \operatorname{Bernoulli}(\kappa), \quad \text { with } \kappa \in(0,1) .
$$

Note that $\mathbf{x}$ has $\mathbb{E}\left[\|\mathbf{x}\|_{0}\right]=\kappa n$ expected spikes thus it is sparse. Then, the matrix $\Psi$ could be regarded as the identity $\mathbb{I}_{n}$, and $\mathbf{x}=\mathbb{I}_{n} \mathbf{w}=\mathbf{w}$. Finally, we take samples using a Gaussian $\Phi$ sensing matrix, i.e., with standard normally distributed entries.
The results, for different sparsity and sampling levels, are shown in Fig. 4. Numerical experiments show that the effective sparsity and $-\|\cdot\|_{1}$ achieve fundamentally the same solution (with effective sparsity, of different parameters $\alpha$ and $\beta$, deliver the same exact solution). The effective sparsity though achieved unintelligible lower error level. Results are therefore promising.

\subsubsection{Power-Law Decaying Signals, e.g., Inverse Problems in Imaging}

The signal $\mathbf{x}$ is now the classical cameraman image, which is sparsified via the Total Variation (TV) [5] and sampled using again a Gaussian matrix $\Phi$.

The results, for a sample rate of 30 percent, are shown in Fig. 5. The numerical experiments show that only the effective sparsity $\|\cdot\|_{1} /\|\cdot\|_{2}$ and $-\|\cdot\|_{1}$ achieve fundamentally the same solution. However, effective sparsity with parameters $\alpha \neq 1$ and $\beta \neq 2$ fail to deliver a correct solution.

It is worth mentioning that, in both previous experiments, tailored optimization algorithms for effective sparsity may achieve enhanced error levels [5], [30]. However, the development of such algorithms is out of our scope.

\subsection{Computing Time of Effective Entropy}

In signals' complexity analysis, of special interest to biomedical sciences [26], the approximate entropy (ApEn) [31] and sample entropy (SampEn) [32] are commonly adopted. Both ApEn and SampEn measures are closely related to the Kolmogorov entropy [33] and suffer from heavy computations. We will compare the computation times of these ApEn and SampEn measures against those required by effective entropy. 


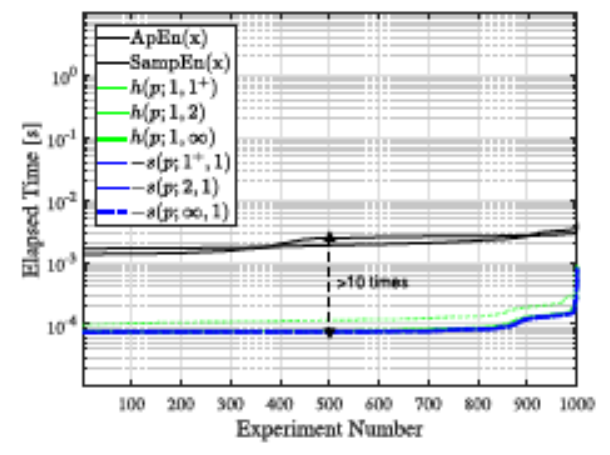

(a) Dimension $n=100$

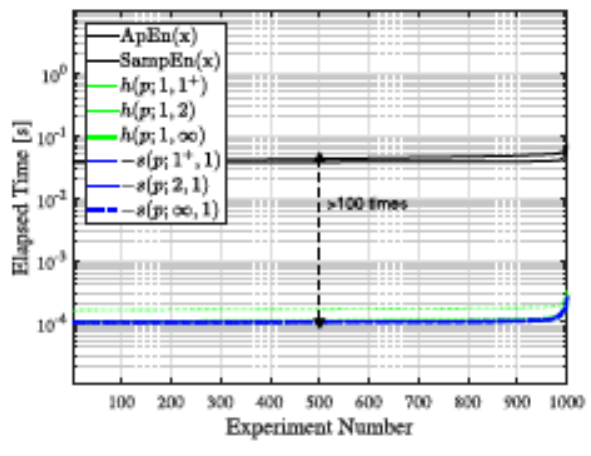

(b) Dimension $n=1000$

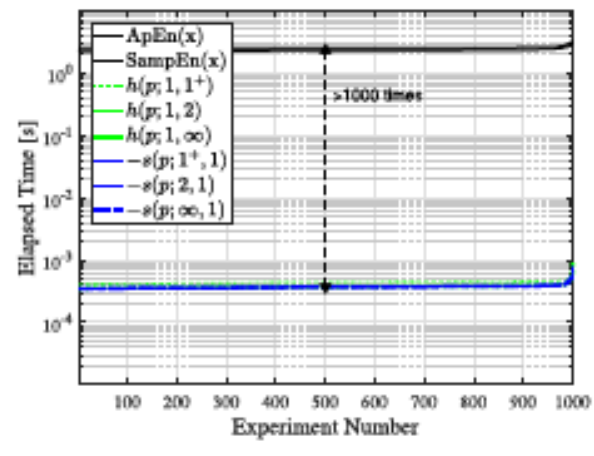

(c) Dimension $n=10000$

Fig. 6. Elapsed time (sorted values) of the complexity analysis of $n$-dimensional binary signals, with fixed 50 percent rate of expected 1 's $(\kappa=0.5$ ). The effective entropy and sparsity achieve approximately $\frac{n}{10}-1$ times smaller computing times than the approximate and sample entropy.

The signal $\mathbf{x}$ under analysis follows (21). Clearly, although $\mathbf{x}$ is artificially generated, this will not alter the performance of complexity ApEn and SampEn measures or of effective entropy. For instance, given parameters $\alpha$ and $\beta$, the computation time of effective entropy is independent of signal's parameter $\kappa$, which determines the uncertainty of the signal. We will compute the effective entropy of the $b$-bins histogram $\mathbf{p} \in[0, n]^{b}$ of $\mathbf{x}$, with $b \approx \sqrt{n}$.

The results, for different dimensions and rate $\kappa=0.5$, are shown in Fig. 6. Numerical experiments show that on average, effective entropy is more than approximately $\frac{n}{10}-1$ times faster than complexity ApEn and SampEn measures. More concretely, effective entropy is a sub-linear time algorithm $\left(\sim O\left(n^{\frac{2}{3}}\right)\right)$ while complexity ApEn and SampEn measures are quadratic $\left(\sim O\left(n^{\frac{3}{2}}\right)\right.$ ) [34]. This efficiency of effective entropy would enable its implementation in wearable devices to perform long-term uninterrupted analyses (see [35] for a real implementation).

It is worth mentioning that, our goal in this Section was to qualify effective sparsity and entropy via standard benchmarks. Nevertheless, several instances of effective sparsity appear in the literature motivated by different purposes and real applications, e.g., see [3], [5]. Some of the most well-known instances will be discussed in the next Section.

\section{Related Work}

We start this last Section presenting decomposability, which is a central property in related works. Let $\mathbf{w}^{1} \oplus \cdots \oplus \mathbf{w}^{G}$ be a partition of $\mathbf{w}$ in $G$ components $\mathbf{w}^{g}$, with $g=1, \ldots, G$. It is said that sparsity is decomposable if there exists an invertible function $\eta: s\left(\Omega_{\mathrm{s}}\right) \rightarrow \mathbb{R}$ and weights $\omega, \omega_{g} \in \mathbb{R}$, such that $\omega \eta \circ s(\mathbf{w})=\sum_{g=1}^{G} \omega_{g} \eta \circ s\left(\mathbf{w}^{g}\right)$. Indeed, decomposability is a very practical property, e.g., in group sparsity [36], but we argue its adoption as an axiom, as it was proposed by [16], [17], and others. The reason is that decomposability imposes a strong condition, consequently producing fewer measures [37]. However, it is a simple exercise to design function $\eta$ and weights $\omega, \omega_{g}$ and show that effective sparsity is decomposable. We can easily verify the following.

\section{Lemma 12 (P12 Decomposability). It holds}

$$
s_{\text {eff }}(\mathbf{w} ; \alpha, \beta)^{\beta}=\sum_{g=1}^{G} \frac{n_{g}\left\langle\mathbf{w}^{g}\right\rangle_{\alpha}^{\beta}}{n\langle\mathbf{w}\rangle_{\alpha}^{\beta}} s_{\text {eff }}\left(\mathbf{w}^{g} ; \alpha, \beta\right)^{\beta} .
$$

Proof. It is a simple verification exercise.

Regarding effective entropy, simply take $\eta()=.{ }^{\alpha}, \omega=1$ and $\omega_{g}=\frac{\left\|\mathbf{p}^{\vartheta}\right\|_{\beta}^{\alpha}}{\|\mathbf{p}\|_{\beta}^{\alpha}}$, to verify its decomposability. Now, we are ready to start the related work discussion.

In the past hundred years, sparsity, traditionally in the form of entropy, has been characterized from the physical or operational, and information theoretic perspectives. Moreover, many instances of effective sparsity appear in the literature, but commonly with one or both parameters fixed. It worth mentioning that, effective sparsity is named $p q$-means in [4], and its origin attributed to [43]. However, [43] suggested its use only with $\alpha \leq 1$ and $\beta=2$ but omitted a derivation. In our literature review then, we categorize the many instances of effective sparsity according to their approach to be qualified as valid sparsity, following the methodology developed by [49]. The related work is summarized in the Table 2 .

The first category, denoted UNK, simply lists measures assessing sparsity but with unknown origins (not easily traceable), such as the min and max ratios and fair utility, appearing in [17], min-max ratio [44], mean log deviation and coefficient of variation [16], and estimator efficiency [50].

The second category, denoted VER, includes measures qualified as sparsity after verification of inherent properties, such as the Rényi entropy (1961) [39] (which verified H1s (symmetry part), $P_{1}, P_{4}, P_{12}$, and joint additivity), Jain fairness (1984) [44], Tsallis entropy (1988) [40], Hoyer sparseness (2004) [45], Bronstein sparseness (2005) [43], max sparsity (2013) [27], and Vershynin effective sparsity (2015) [14].

A third and more interesting category, denoted $O P E$, includes measures designed to have operational meaning with physical basis, such as the Hartley entropy counting the possible symbols in a sequence of arbitrary systems (1928) [38]; Simpson and Gini-Simpson diversities measuring the probability that two randomly chosen individuals from a population belong to the same group (1949) [46]; Atkinson inequality using principles of ranking of distributions (1970) [41]; Berger-Parker dominance measuring the numerical importance of the most abundant species (1970) [48]; Hill diversity estimating the effective number of species in a sample (1973) [42]; collision entropy measuring the probability that a random variable takes on the same value twice in two independent experiments (1995) [47], min entropy which operational meaning is studied in the context of quantum 
TABLE 2

Summary of the Literature Review

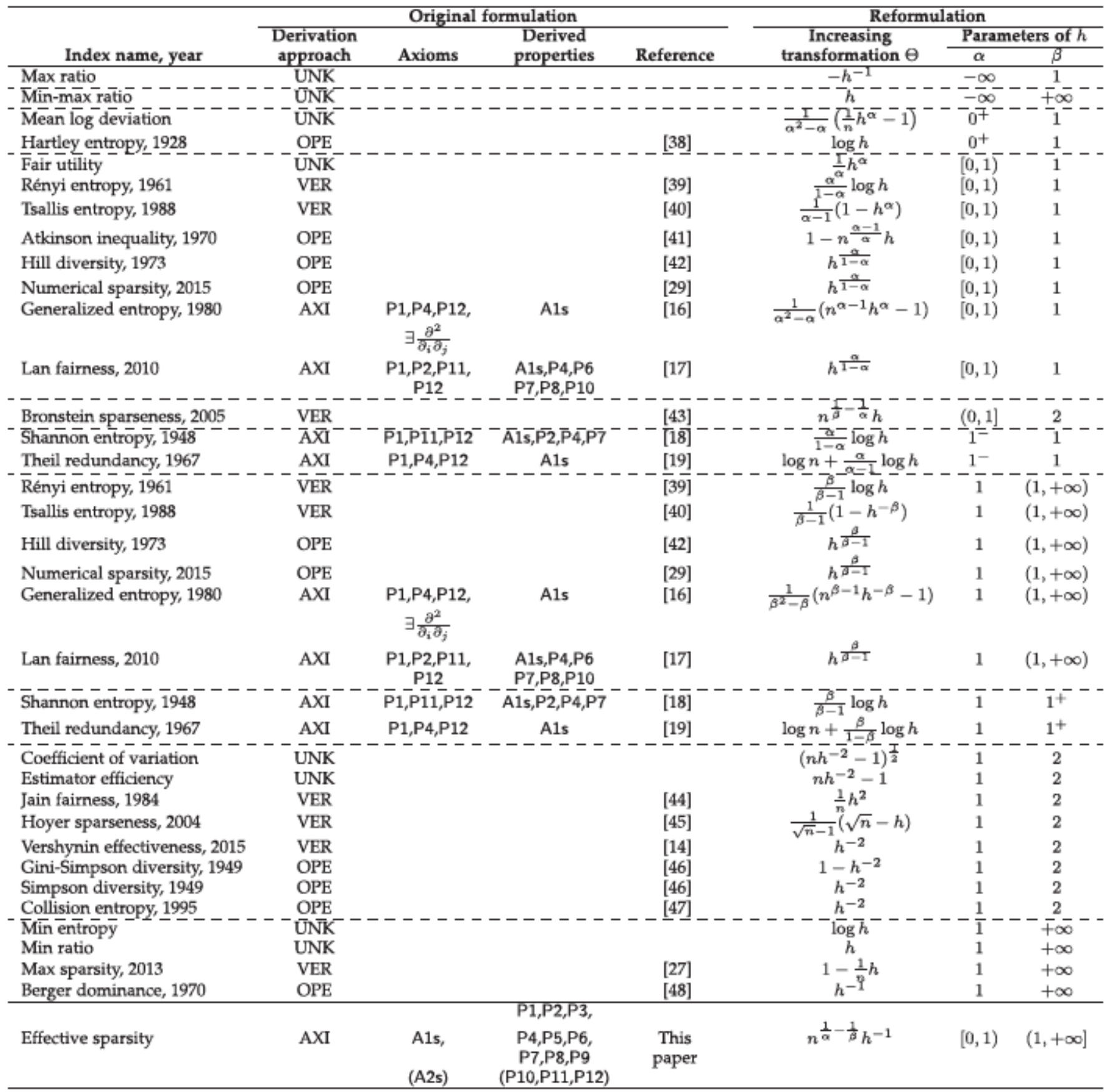

Classical and more recent measures are reuritten in terms of effective entropy $h_{\text {off }}(\cdot ; \alpha, \beta)=h$ and increasing transformations $\Theta$, Lemma 9 . In the Table, Ax denotes axiom, Px property, $\frac{\partial^{2}}{\partial_{2} \partial_{j}}$ second order differentiability, and $n$ is the dimension.

systems (2009) [51]; and numerical sparsity establishing an analogy with the Rényi entropy (2015) [29].

The last and more mathematically rigorous category, denoted AXI, indudes measures derived from an axiomatic approach or first principles, such as the Shannon entropy (1948) [18] (postulated $P_{1}, P_{11}$ and $P_{12}$ as axioms, and verified A1s, $P_{2}, P_{4}, P_{7}$, and joint additivity as properties), Theil redundancy (1967) [19], Generalized entropy (1980) [16], and Lan fairness (2010) [17]. Further details on the so-called axioms or properties in this category, are presented in Table 2. In the Table, columns 3-4 indicate the proposed axioms and derived properties. Interestingly, all works under the $A X I$ category share several common aspects. All of these works assumed H1s, and proposed $\mathrm{P}_{1}$ and $\mathrm{P}_{12}$ as axioms. Also, from $\mathrm{H} 1 \mathrm{~s}$ and multiple postulated axioms (including $\mathrm{P}_{1}$ and $\left.P_{12}\right)$, all of these works derived A1s, Dalton's first law of inequality, but as a property. This paper belongs to this category, and similarly to the works here, we assume H1s. However, contrary to those works, we prove that, A1s completely characterizes sparsity. In this sense, what we propose is a minimal or effective characterization of sparsity. For instance then, continuity (P1) does not need to be postulated as an axiom any longer. Although, this was expected because $A 1 s$ has a differentiability angle. 
Nevertheless, similar to related work in the OPE category, this paper also develops an operational meaning. Indeed, following novel ideas from sparse recovery [14], we study the geometry and operational meaning of our derived effective sparsity. Furthermore, this paper also enables a simplified verification test for measures in the VER category. Now, under the requirements of Theorem 3, the Gini coefficient is now qualified as sparsity by simply verification of A1s, instead of evaluating various other properties [4].

We finish the Section with an analysis of measures proposed in related work, and which were independently developed in different fields and periods. These measures are listed in Table 2 and reformulated in terms of effective entropy, which facilitates the reformulation because it does not carry the dimension-dependent scale factor. Note that the reformulations indude two components. First, in columns 78 , the parameters $\alpha$ and $\beta$ of effective entropy. Second, in column 6 , an increasing transformation $\Theta$ that preserves the sparsity ordering, Lemma 9. Such transformations include the logarithm, positive affine, power and double inverse $-\frac{1}{x}$ functions, or compositions of them, cf. [6], [7]. For instance, for the Hartley entropy $\Theta=\log$ and parameters $\alpha=0^{+}$and $\beta=1$. Moreover, these different transformations may have been originally introduced to enable specific properties or operational meaning. For instance, for $\beta=1$ fixed, the power function $(.)^{\frac{\alpha}{\alpha-1}}$, which appears in many measures, serves to normalize the range from $\left[1, n^{\bar{\alpha}} \bar{\beta}\right]$ to $(0,1]$. Actually, the range of effective sparsity and effective entropy are $\left[1, n^{\frac{1}{\alpha}} \frac{1}{\beta}\right]$ and $\left[1, b^{\frac{1}{2}} \frac{1}{p}\right]$, respectively; and both could be easily normalized to $(0,1]$ dividing them by the scaling factors $n^{\frac{1}{\alpha}} \frac{1}{\beta}$ and $b^{\frac{1}{\alpha}} \frac{1}{\beta}$, also respectively. Finally, these reformulations allow us to also recognize other similarities between the measures. For instance, the numerical sparsity [29] in information theory (2015), equals Lan fairness [17] in communication networks (2010), Hill diversity [42] in ecology (1973), and so on. In brief, numerous classical measures can be reformulated in terms of effective entropy, such as the generalized entropy [16] and its special cases, including the Shannon entropy.

\section{Conclusion}

In the past hundred years, the measures to compute sparsity, traditionally in the form of entropy, have been characterized and qualified from the physical or operational, and information theoretic perspectives. In this paper, instead, a classical mathematical approach using equivalence relations has enabled a minimal characterization of sparsity. We have introduced and demonstrated the generalized convexity nature of sparsity. This key property has motivated the functional form of sparsity, which conceptually unifies quotients of functions that have been continuously observed in the literature.

Lastly, sparsity is a central notion in modern sensing, pattern recognition and communications systems, which aim to exploit the compact representations of underlying real-world phenomena. Granted, the possibilities for future research from this paper are extensive. An extension of effective entropy to relative and conditional entropy would be desirable. Further extensions could indude the quantum setting. Moreover, effective entropy is a sub-linear time algorithm and should enable large datasets analysis. It would also be of interest to explore how effective sparsity can be efficiently optimized in sparse recovery algorithms. To that end, the work presented in this paper suggests new insights on the dynamics, properties and geometry of sparsity that may be of benefit to the design of algorithms.

\section{ACKNOWLEDGMENTS}

G. Pastor would like to thank Ilkka Norros (formerly at VTT Technical Research Centre of Finland), Edward Mutafungwa (Aalto University) and Peter Bryant (IE Business School) for their careful reading and comments on the paper. Authors also acknowledge the reviewers' suggestions which improved the presentation of contributions. The work of G. Pastor was supported in part by the Academy of Finland under Grant 317432, and the Helsinki Institute for Information Technology.

\section{References}

[1] E J. Candes and T. Tao, "Decoding by linear programming," IEEE Trans. Inf. Theory, vol. 51, no. 12, pp. 4203-4215, Dec. 2005.

[2] M. Malek-Mohammadi, A. Koochakzadeh, M. Babaie-Zadeh, M. Jansson, and C. R. Rojas, "Successive concave sparsity approximation for compressed sensing," IEEE Trans. Signal Process., vol. 64, no. 21, pp. 5657-5671, Nov. 2016.

[3] M. Pilanci, L. El Ghaoui, and V. Chandrasekaran, "Recovery of sparse probability measures via convex programming," in Proc. 25 th Int. Conf. Neural Inf. Process. Syst., 2012, pp. 2420-2428.

[4] N. Hurley and S. Rickard, "Comparing measures of sparsity," IEEE Trans. Inf. Theory, vol. 55, no. 10, pp. 4723-4741, Oct. 2009.

[5] P. Yin, E Esser, and J. Xin, "Ratio and difference of L1 and L2 norms and sparse representation with coherent dictionaries," Commun. Inf. Syst., vol. 14, no. 2, pp. 87-109, 2014.

[6] A. Maronidis, E. Chatzilari, S. Nikolopoulos, and I. Kompatsiaris, "A generalised differential sparsity measure for reconstructing compressively sampled signals," Digit. Signal Process., vol. 74, pp. 14-29, 2018.

[7] S. Huang and T. D. Tran, "Sparse signal recovery via generalized entropy functions minimization," IEEE Trans. Signal Process., vol. 67 , no. 5, pp. 1322-1337, Mar. 2019.

[8] F. M. Zennaro and K. Chen, "Towards understanding sparse filtering: A theoretical perspective," Neural Netw., vol. 98, pp. 154-177, 2018.

[9] G. Mary P. D., B. Madathil, and S. N. George, "Entropy-based reweighted tensor completion technique for video recovery," IEEE Trans. Circuits Syst. Video Technol., vol. 30, no. 2, pp. 415-426, Feb. 2020.

[10] S. Zhang, Y. Liu, and X. Li, "Autofocusing for sparse aperture ISAR imaging based on joint constraint of sparsity and minimum entropy," IEEE J. Sel. Topics Appl. Earth Observ. Remote Sens., vol. 10, no. 3, pp. 998-1011, Mar. 2017.

[11] S. Skiena, Implementing Discrete Mathematics - Combinatorics and Graph Theory With Mathematica. Reading, MA, USA: AddisonWesley, 1990.

[12] H. J. Greenberg and W. P. Pierskalla, "A review of quasi-convex functions," Operations Res., vol. 19, no. 7, pp. 1553-1570, 1971.

[13] A. Cambini and L. Martein, General ized Convexity and Optimization: Theory and Applications. Berlin, Germany: Springer, 2009.

[14] R. Vershynin, Estimation in High Dimensions: A Geometric Perspective. Berlin, Germany: Springer, 2015, pp. 3-66.

[15] H. Dalton, "The measurement of the inequality of incomes," Econ. J., vol. 30, no. 119 , pp. 348-361, Sep. 1920.

[16] A. F. Shorrocks, "The class of additively decomposable inequality measures," Econometrica, vol. 48, no. 3, pp. 613-625, Apr. 1980.

[17] T. Lan, D. Kao, M. Chiang, and A. Sabharwal, "An axiomatic theory of fairness in network resource allocation," in Proc. IEEE Int. Conf. Comput. Commun., 2010, pp. 1-9.

[18] C E. Shannon, "A mathematical theory of communication," Bell Syst. Tech. J., vol. 27, no. 3, pp. 379-423, Jul. 1948.

[19] $\mathrm{H}$. Theil, Economics and Information Theory. Amsterdam, The Netherlands, North Holland, 1967.

[20] A. Blake, "A Boolean derivation of the Moore-Osgood theorem," J. Symbolic Logic, vol. 11, no. 3, pp. 65-70, Sep. 1946. 
[21] J. Martin, G. Mayor-Forteza, and J. Suner, "On dispersion measures," Mathware Soft Comput., vol. 8, no. 3, pp. 227-237, 2001.

[22] S. M. Khorasani, G. A. Hodtani, and M. M. Kakhki, "Effects of preprocessing on the ECG signal sparsity and compression quality," in Proc. 8th Int. Conf. Comput. Knowl. Eng., 2018, pp. 268-273.

[23] M. D. Esteban and D. Morales, "A summary on entropy statistics," Kybermetika, vol. 31, no. 4, pp. 337-346, 1995.

[24] P. S. Bullen, Handbook of Means and Their Inequalities. Dordrecht, Netherlands: Springer, 2003.

[25] G. Liu and W. Zhang, "Recovery of future data via convolution nudear norm minimization," 2019, arXiv e-prints: 1909.03889.

[26] Z. Liang et al. "EEG entropy measures in anesthesia," Front. Comput. Neurosci., vol. 9, Feb. 2015, Art. no. 16.

[27] G. Pastor, I. Mora-Jimenez, R. Jantti, and A. J. Caamano, "Sparsitybased criteria for entropy measures," in Proc. IEEE Int. Symp. Wireless Commun. Syst., 2013, pp. 1-5.

[28] M. Petrovici, C. Damian, and D. Coltuc, "Image reconstruction from incomplete measurements: Maximum entropy versus L1 norm optimization," in Proc. Int. Symp. Signals Circuits Syst., 2017, pp. 1-4.

[29] M. E. Lopes, "Unknown sparsity in compressed sensing: Denoising and inference," IEEE Trans. Inf. Theory, vol. 62, no.9, pp. 5145-5166, 2016.

[30] G. Pastor, "A low-rank tensor model for imputation of missing vehicular traffic volume," IEEE Trans. Veh. Technol., vol. 67, no. 9, pp. 8934-8938, Sep. 2018.

[31] S. M. Pincus, "Approximate entropy as a measure of system complexity," Proc. Nat. Acal. Sci. USA, vol. 88, no. 6, pp. 2297-2301, Mar. 1991.

[32] J.S. Richman and J. R. Moorman, "Physiological time-series analysis using approximate entropy and sample entropy," Amer. J. Physiol. Heart Circul. Physid., vol. 278, no. 6, pp. H2039-H2049, Jun. 2000.

[33] Y. G. Sinai, "On the notion of entropy of a dynamical system," Doklady Russian Acad. Sci., vol. 124, pp. 768-771, 1959.

[34] Y. H. Pan, Y. H. Wang, S. F. Liang, and K. T. Lee, "Fast computation of sample entropy and approximate entropy in biomedicine," Comput. Methods Programs Biomed., vol. 104, no. 3,pp. 382-396, Dec. 2011.

[35] M. I. Chidean, G. Pastor, E. Morgado, J. Ramiro-Bargueño, and A. J. Caamaño, "Wireless sensor network for low-complexity entropy determination of human gait," in Proc. IEEE Int. Symp. Pers. Indoor Mobile Radio Commun., 2013, pp. 2644-2648.

[36] M. Yuan and Y. Lin, "Model selection and estimation in regression with grouped variables," J. Roy. Statist. Soc. Ser. Statist. Methodol., vol. 68 , no. 1, pp. 49-67, 2006

[37] F. A. Cowell, "Theil, inequality and the structure of income distribution," Suntory and Toyota International Centres for Economics and Related Disciplines, LSE, no. 67, May 2003.

[38] R. V. L Hartley, "Transmission of information," Bell Syst. Tech. J., vol. 7, no. 3, pp. 535-563, Jul. 1928.

[39] A. Renyi, "On measures of entropy and information," in Proc. 4th Berkeley Symp. Math. Statist. Probability, 1961, pp. 547-561.

[40] C. Tsallis, "Possible generalization of Boltzmann-Gibbs statistics," J. Statist. Phys., vol. 52, no. 1, pp. 479-487, Jul. 1988.

[41] A. B. Atkinson, "On the measurement of inequality," J. Econ. Theory, vol. 2, no. 3, pp. 244-263, Sep. 1970.

[42] M. O. Hill, "Diversity and evenness: A unifying notation and its consequences," Ecology, vol. 54, no. 2, pp. 427-432, Mar. 1973.

[43] A. M. Bronstein, M. M. Bronstein, M. Zibulevsky, and Y. Y. Zeevi, "Sparse ICA for blind separation of transmitted and reflected images," Int.J. Imag. Syst. Technol., vol. 15, no. 1, pp. 84-91,Jul. 2005.

[44] R. Jain, D. M. Chiu, and W. R. Hawe, "A quantitative measure of faimess and discrimination for resource allocation in shared computer system," Eastem Res. Lab., Digit. Equip. Corporation, Maynard, MA, USA, Tech. Rep. DEC-TR-301, Sep. 1984.

[45] P. O. Hoyer, "Non-negative matrix factorization with sparseness constraints," J. Mach. Larm. Res., vol. 5, pp. 1457-1469, Dec. 2004.

[46] E. H. Simpson, "Measurement of diversity," Nature, vol. 163, no. 4148, Apr. 1949, Art. no. 688

[47] C. H. Bennett, G. Brassard, C. Crepeau, and U. M. Maurer, "Generalized privacy amplification," IEEE Trans. Inf. Theory, vol. 41, no. 6, pp. 1915-1923, Nov. 1995.

[48] W. H. Berger and F. L. Parker, "Diversity of planktonic foraminifera in deep-sea sediments," Science, vol. 168, no. 3937, pp. 1345-1347, Jun. 1970.

[49] I. Csiszar, "Axiomatic characterizations of information measures," Entropy, vol. 10, no. 3, pp. 261-273, 2008.

[50] M. G. Kendall and A. Stuart, Kendall's Advanced Theory of Statistics, vol. 2. New York, NY, USA: Hafner, 1961.
[51] R. Konig, R. Renner, and C. Schaffner, "The operational meaning of min- and max-entropy," IEEE Trans. Inf. Theory, vol. 55, no. 9, pp. 4337-4347, Sep. 2009.

[52] P. Cheridito and E. Kromer, "Reward-risk ratios," J. Investment Strategies, vol. 3, pp. 3-18, Dec. 2013.

[53] L Yuan and H. K. Kesavan, "Minimum entropy and information measure," IEEE Trans. Syst., Man, Cybern. C, Appl. Rev., vol. 28, no. 3, pp. 488-491, Aug. 1998.

[54] N. R. Pal and J. C. Bezdek, "Measuring fuzzy uncertainty," IEEE Trans. Fuzzy Syst., vol. 2, no. 2, pp. 107-118, May 1994.

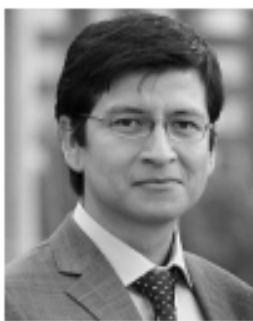

Giancarlo Pastor received the BSc degree in electronics engineering from the Pontifical University Catholic of Peru, Lima, Peru, the BSc degree in mathematics from the Autonomous University of Madrid, Madrid, Spain, and the PhD degree in electrical engineering from the Aalto University, Espoo, Finland, Carlos III University of Madrid, Madrid, Spain, and King Juan Carlos University, Madrid, Spain, with mention in Innovation and Entrepreneurship by EIT Digital. $\mathrm{He}$ is a postdoctoral researcher with Aalto University, Finland, and an adjunct professor with IE Business School, Spain. His research focuses on clustering and imputation of high-dimensional data.

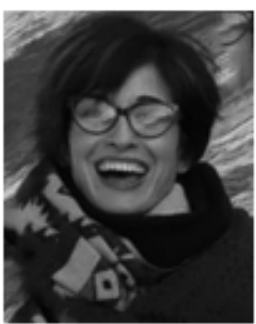

Inmaculada Mora-Jiménez received the degrøe in telecommunication engineering from the Polytechnic University of Valencia, Valencia, Spain, and the $\mathrm{PhD}$ degree in telecommunication engineering from the Carlos III University of Madrid, Madrid, Spain. She is currently an associate professor with the Department of Signal Theory and Communications, Telematics and Computing Systems, King Juan Carlos University, Spain. She is co-author of 40JCRindexed papers and has participated in 18 competitive research projects ( $\mathrm{PI}$ of 4 ). Her research focuses on data science and machine learning with application to image processing, bioengineering, and communications.

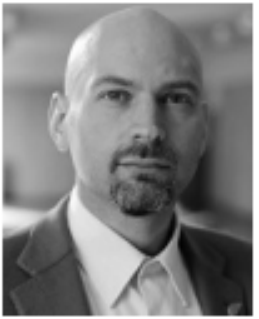

Riku Jäntti (Senior Member, IEEE) received the MSc (with distinction) degree in electrical engineering and the DSc (with distinction) degree in automation and systems technology, both from the Helsinki University of Technology (TKK), Espoo, Finland, in 1997 and 2001. He is a full professor of communications engineering and the head of the Department of Communications and Networking, Aalto University School of Electrical Engineering, Finland. Prior to joining Aalto University (formerly known as TKK) in August 2006 , he was professor pro tem with the Department of Computer Science, University of Vaasa. He is an associate editor of the IEEE Transactions on Vehicular Technology. He is also IEEE VTS distinguished lecturer (Class 2016). His research interests include machine type communications, cloud based radio access networks, backscatter communications, quantum communications, and radio frequency inference.

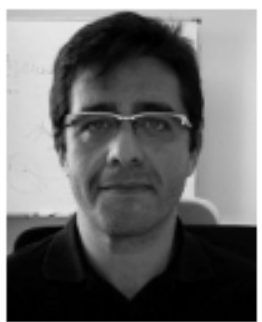

Antonio J. Caamaño received the BSc and MSc degrees in theoretical physics from the Autonomous University of Madrid, Madrid, Spain, in 1995 , and the $\mathrm{PhD}$ degree in telecommunications engineering from the Carlos III University of Madrid, Madrid, Spain, in 2003. Currently, he is a professor with the Department of Signal Theory and Communications, Telematics and Computing Systems, King Juan Carlos University, Spain. His research focuses on large-scale MANET optimization, distributed computing, and processing of biological signals.

$\triangleright$ For more information on this or any other computing topic, please visit our Digital Library at www.computer.org/csdl. 